\title{
UNIFORM CROSS NORMS AND TENSOR PRODUCTS OF BANACH ALGEBRAS ${ }^{1}$
}

\author{
BY JESỨS GIL DE LAMADRID \\ Communicated by Walter Rudin, July 22, 1963
}

1. Introduction. The present work grew out of an attempt to answer three questions. The first two were raised by B. R. Gelbaum [1]. One is: Which cross norms $\alpha$ of the algebraic tensor product $A \otimes B$ of two Banach algebras $A$ and $B$ are compatible with multiplication? Compatibility with multiplication means that

$$
\alpha\left(\mathrm{t}_{1} \mathrm{t}_{2}\right) \leqq \alpha\left(\mathrm{t}_{1}\right) \alpha\left(\mathrm{t}_{2}\right),
$$

for every two tensors $\mathrm{t}_{1}, \mathrm{t}_{2} \in A \otimes B$. The second question is: Is the socalled least cross norm $\lambda$, in particular, compatible with multiplication? The third question was raised by B. R. Gelbaum and the author in [2]. It is: Given two Banach spaces $E$ and $F$ each of which has a Schauder basis, for what cross norms $\alpha$ do the resulting complete tensor products $E \otimes_{\alpha} F$ have Schauder bases?

The present state of knowledge concerning the first two questions is as follows. In [3] Gelbaum (see also Tomiyama [4]) defined on the algebraic tensor product $A \otimes B$ of two Banach algebras a multiplication which is the linear extension of the following multiplication on decomposable tensors.

$$
\left(U_{1} \otimes V_{1}\right)\left(U_{2} \otimes V_{2}\right)=U_{1} U_{2} \otimes V_{1} V_{2},
$$

where $U_{1}, U_{2} \in A$ and $V_{1}, V_{2} \in B$. Under this multiplication $A \otimes B$ becomes a complex algebra. The greatest cross norm $\gamma$ on $A \otimes B$ is compatible with this multiplication, which can be extended to $A \otimes_{\gamma} B$, turning the latter into a Banach algebra. It is not known, in general, whether the tensor product $A \otimes_{\gamma} B$ of two semisimple commutative Banach algebras is semisimple, although this is the case for all known concrete cases. No single cross norm, of a general character and known to be different from $\gamma$, has been found that is compatible with multiplication. Gelbaum has shown that the nuclear (trace) norm of Grothendieck, which Gelbaum derived independently, is compatible with multiplication, but no single instance is known where that norm differs from $\gamma$. In particular, little seems to be known about the compatibility with multiplication of the least cross norm $\lambda$ of $A \otimes B$.

In the present work we exhibit a broad class of cross norms, includ-

${ }^{1}$ Support of the present work by a National Science Foundation research grant (NSF G-19752) is gratefully acknowledged. 
ing many of general character, which are compatible with multiplication. A cross norm is said to be of "general character" (according to Schatten) if it is given by an abstract definition which applies to all tensor products of Banach spaces. In this sense, both $\lambda$ and $\gamma$ are of general character. It is shown, at least when all algebras involved have identity elements, that to every uniform cross norm (in the sense of Schatten [5]) of $A \otimes B$, not necessarily compatible with multiplication, there corresponds a cross norm (its associated cross norm) which is compatible with multiplication. Concerning the second question we exhibit examples in which $\lambda$ is compatible with multiplication, and examples in which it is not. We also give sufficient conditions for such a compatibility.

The present state of knowledge concerning the third question is as follows. In [2] it was shown that Schauder bases of two given Banach spaces $E$ and $F$ define a system which serves as a basis for both $E \otimes_{\lambda} F$ and $E \otimes_{\gamma} F$. Each one of these two tensor products had to be treated separately and the arguments were rather involved. The methods employed do not generalize to any other tensor products. No other cross norm $\alpha$ of a general character is known to have the property that the existence of Schauder bases of both $E$ and $F$ implies the existence of a Schauder basis for $E \otimes_{\alpha} F$. In the present work we show that every uniform cross norm has such a property.

During the development of this work, as well as during many earlier occasions the author had the benefit of many illuminating conversations with B. R. Gelbaum.

2. The Kronecker product. Let $E$ and $F$ be two Banach spaces, and $U: E \rightarrow E$ and $V: F \rightarrow F$ be bounded linear operators. The Kronecker product of $U$ and $V$ is the operator $U \otimes V$ defined by the relation

$$
U \otimes V(x \otimes y)=U x \otimes V y,
$$

for $x \in E$ and $y \in F$. This notion generalizes the classical notion of Kronecker product of matrices. Let $\alpha$ be a cross norm of the algebraic tensor product $E \otimes F$. With Schatten [5] we say that $\alpha$ is a uniform cross norm if for every tensor $\mathrm{t} \in E \otimes F$, given by

$$
\mathrm{t}=\sum_{i=1}^{n} x_{i} \otimes y_{i}
$$

for $x_{i} \in E$ and $y_{i} \in F$, we have

$$
\alpha[U \otimes V(\mathrm{t})]=\alpha\left[\sum_{i=1}^{n} U x_{i} \otimes V y_{i}\right] \leqq\|U\|\|V\| \alpha(\mathrm{t}),
$$

for every $U \in B(E)$, the algebra of all bounded linear operators of $E$ 
into itself and every $V \in \Theta(F)$. If $\alpha$ is a uniform cross norm of $E \otimes F$ and $U \in B(E)$ and $V \in B(F)$, then clearly the Kronecker product defined on $E \otimes F$ by (2.1) can be extended to an operator $U \otimes V$ of the complete tensor product $E \otimes_{\alpha} F$ into itself. We call this extended operator also the Kronecker product (with respect to $\alpha$ when there is any possible ambiguity) of $U$ and $V$.

THEOREM 2.1. Let $A$ be a subalgebra of $B(E)$ and $B$ a subalgebra of $\Theta(F)$. Suppose that $\alpha$ is a uniform cross norm of $E \otimes F$. Then the Kronecker product defines an algebraic isomorphism of the algebra $A \otimes B$ onto a subalgebra of $B\left(E \otimes_{\alpha} F\right)$.

The subalgebra of $\Theta\left(E \otimes_{\alpha} F\right)$ isomorphic to $A \otimes B$ in Theorem 2.1 is called the Kronecker product of $A$ and $B$. We denote by $\bar{\alpha}$ the operator norm of $B\left(E \otimes_{\alpha} F\right)$. We also use this symbol to denote the norm defined on the Kronecker product of $A$ and $B$ by the operator norm of $B\left(E \otimes_{\alpha} F\right)$. By Theorem 2.1 this norm in turn defines a norm on $A \otimes B$, which we continue to denote by $\bar{\alpha}$.

THEOREM 2.2. The norm $\bar{\alpha}$ on $A \otimes B$ is a cross norm which is compatible with multiplication. Hence $A \otimes_{\bar{\alpha}} B$ is a Banach algebra.

3. Tensor products of arbitrary Banach algebras. We now extend the results of the previous section to arbitrary Banach algebras $A$ and $B$. We do this by considering these algebras as algebras of operators over themselves. This, however, is not always possible in the absence of identity elements. The following constructions represent a compromise which covers most of the interesting cases. By an approximate right identity of $A$ we mean a subset $N$ of the solid unit sphere of $A$ with the property that every $U \in A$ belongs to the closure $\mathrm{Cl}(U N)$ of the set $U N$. Now, if $A$ has no approximate right identity, let $E$ be the smallest Banach algebra with identity containing $A$ isometrically. If $A$ has an approximate right identity, we let $E=A$. We follow a similar convention with respect to the symbols $B$ and $F$. We then have that $A$ can be considered as a closed subalgebra of $B(E)$ by means of its left regular representation, and similarly for $B$ and $B(F)$. Consequently, a uniform cross norm of $E \otimes F$ induces a cross norm $\bar{\alpha}$ on $A \otimes B$, by the methods of the previous section, which is compatible with multiplication in $A \otimes B$. In this way $A \otimes_{\bar{\alpha}} B$ becomes a Banach algebra. We study some properties of this algebra which center about the concept of semisimplicity. The discussion is based on the following two theorems which are generalizations of theorems of Gelbaum $[1 ; 3]$.

Let $E$ and $F$, for the moment, denote arbitrary Banach spaces. An ordinary norm $\alpha$ of $E \otimes F$ is one satisfying the relation $\lambda \leqq \alpha \leqq \gamma$, where 
$\lambda$ is the least cross norm [5] of $E \otimes F$. An ordinary norm is ipso facto a cross norm. Let $E^{\prime}$ denote the dual (conjugate) space of $E$ and similarly for $F$ and $F^{\prime}$. If $x \in E$ and $x^{\prime} \in E^{\prime}$, we denote by $\left\langle x, x^{\prime}\right\rangle$ the action of $x$ and $x^{\prime}$ on each other. Let $\alpha$ be an ordinary norm of $E \otimes F$. It follows from the work of Schatten [5] that the algebraic tensor product $E^{\prime} \otimes F^{\prime}$ can be imbedded in $\left(E \otimes_{\alpha} F\right)^{\prime}$. We write $E^{\prime} \otimes F^{\prime} \subset\left(E \otimes_{\alpha} F\right)^{\prime}$. The action of a tensor $x^{\prime} \otimes y^{\prime} \in E^{\prime} \otimes F^{\prime}$ on $\mathrm{t} \in E \otimes F$, given by (2.2), is given by

$$
\left\langle\mathrm{t}, x^{\prime} \otimes y^{\prime}\right\rangle=\sum_{i=1}^{n}\left\langle x_{i}, x^{\prime}\right\rangle\left\langle y_{i}, y^{\prime}\right\rangle
$$

Let $A$ be any Banach algebra. We denote by $M_{A}$ the space of all bounded multiplicative linear functionals on $A$ under the weak* topology.

THEOREM 3.1. Let $A$ and $B$ be two arbitrary Banach algebras and $\beta$ an ordinary norm of $A \otimes B$ which is compatible with multiplication. Then, for $x^{\prime} \in M_{A}$ and $y^{\prime} \in M_{B}$, the assignment $\left(x^{\prime}, y^{\prime}\right) \rightarrow x^{\prime} \otimes y^{\prime}$ maps the space $M_{A} \times M_{B}$ homeomorphically onto $M_{A \otimes_{\beta} B}$.

Let $E$ and $F$ be, again for the moment, two arbitrary Banach spaces, and $\alpha$ an ordinary norm of $E \otimes F$. Now both $E \otimes_{\alpha} F$ and $E \otimes_{\lambda} F$ contain the algebraic tensor product $E \otimes F$. Because of the relation $\alpha \geqq \lambda$ the identity mapping, considered as a mapping of $E \otimes F$ into $E \otimes_{\lambda} F$, can be extended to a bounded linear transformation $\Phi_{\lambda}^{a}: E \otimes_{\alpha} F \rightarrow E \otimes_{\lambda} F$. It seems to be extremely difficult, in general, to determine whether $\Phi_{\lambda}^{a}$ is one-to-one. If it is, we say that $\alpha$ is a faithful cross norm.

Theorem 3.2. Let $A$ and $B$ be commutative semisimple Banach algebras and $\beta$ an ordinary norm of $A \otimes B$ which is compatible with multiplication. Then $A \otimes_{\beta} B$ is semisimple if and only if $\beta$ is a faithful cross norm.

Let now $A$ and $E$ and $B$ and $F$ be related as described at the beginning of this section and suppose that $\alpha$ is a uniform cross norm of $E \otimes F$. The application of the above two theorems to $\bar{\alpha}$ is made possible by the following theorem.

THEOREM 3.3. If $\alpha$ is an ordinary norm of $E \otimes F$, then $\bar{\alpha}$ is an ordinary norm. If $\alpha$ is faithful then $\bar{\alpha}$ is faithful.

Corollary 3.1. Let $A$ and $B$ be two commutative semisimple Banach algebras, and $\alpha$ a uniform faithful cross norm of $E \otimes F$. Then $A \otimes_{\bar{\alpha}} B$ is a semisimple commutative Banach algebra. 
4. Examples and further properties. The least cross norm $\lambda$ of $E \otimes F$, which extends the least cross norm of $A \otimes B C E \otimes F$, is uniform [5, p. 30, Lemma 2.6]. It follows that $\bar{\lambda}$ is compatible with multiplication. Since $\lambda$ is clearly a faithful cross norm, it follows that the tensor product $A \otimes_{\bar{\lambda}} B$ of two commutative semisimple Banach algebras is a semisimple Banach algebra.

We say that a uniform cross norm $\alpha$ of $E \otimes F$ is self-associated if $\alpha=\bar{\alpha}$ on $A \otimes B \subset E \otimes F$.

THEOREM 4.1. If $\alpha$ is a uniform cross norm on $E \otimes F$, which is compatible with multiplication on $E \otimes F$, then $\alpha$ is self-associated.

It turns out, a nontrivial fact, that the greatest cross norm $\gamma$ of $E \otimes F$ is an extension of the greatest cross norm of $A \otimes B C E \otimes F$. Since $\gamma$ is compatible with multiplication on $E \otimes F$, then, by Theorem 4.1, $\gamma$ is self-associated and we can write $\gamma=\bar{\gamma}$. A similar discussion is valid for the nuclear norm of Grothendieck [6]. For another discussion of this norm see [7], where it is called the trace norm.

5. The least cross norm $\lambda$. We now discuss the second of the two questions that motivated this study: given two Banach algebras $A$ and $B$, is the least cross norm $\lambda$ of $A \otimes B$ compatible with multiplication?

THEOREM 5.1. If either $A$ or $B($ say $A)$ is an algebra of functions (i.e., the Gelfand representation is isometric), then $\lambda$ is compatible with multiplication in $A \otimes B$, and $A \otimes_{\lambda} B$ is the corresponding algebra of $B$-valued mappings. $A \otimes_{\lambda} B$ is commutative and semisimple if $B$ is commutative and semisimple.

The least cross norm is not always compatible with multiplication, as the following example shows. Let $A=l_{1}$, the algebra of absolutely summable complex sequences with convolution as multiplication. In such a sequence we shall only indicate terms which might not be zero. For instance $U=\left(\delta_{0}, \delta_{1}\right)$ stands for an element of $l_{1}$, with $\delta_{i}=0$, for $i>1$. It follows from a theorem of Grothendieck [6, p. 90, Example 2] that $l_{1} \otimes_{\lambda} B$ can be identified with the space of all unconditionally summable sequences of elements of $B$. In [7, p. 30, Lemma 2] we have computed $\lambda$ in this case and we refer the reader to that article in connection with the computations that follow. The multiplication on $A \otimes B$ defined by (1.2) above becomes, in the case of $l_{1} \otimes B$, convolution of sequences of elements in $B$. We now specialize $B$ to be $l_{1}$ also and show that $\lambda$ is not compatible with convolution in $l_{1} \otimes l_{1}$. Let $V_{0}=(1,-1)$ and $V_{1}=(1,1) \in l_{1}=B$, and $\mathrm{t}=\left(V_{0}, V_{1}\right) \in l_{1} \otimes l_{1}$ $=A \otimes B$. Now 


$$
\lambda(t)=\max \left(\left|\epsilon_{0}-\epsilon_{1}\right|+\left|\epsilon_{0}+\epsilon_{1}\right|\right)=2,
$$

where the max is taken for $-1 \leqq \epsilon_{0}, \epsilon_{1} \leqq 1$. On the other hand

$$
\begin{aligned}
\lambda(\mathrm{t} * \mathrm{t})= & \lambda\left(V_{0} * V_{0}, 2 V_{0} * V_{1}, V_{1} * V_{1}\right) \\
= & \max \left[\left(\delta_{0}+\delta_{1}\right) \epsilon_{0}-2 \delta_{0} \epsilon_{1}-\left(\delta_{1}-\delta_{0}\right) \epsilon_{2}\right. \\
& \left.+\left(\delta_{1}+\delta_{2}\right) \epsilon_{0}+2 \delta_{2} \epsilon_{1}-\left(\delta_{1}-\delta_{2}\right) \epsilon_{2}\right] \\
\geqq & 8>[\lambda(\mathrm{t})]^{2}=4 .
\end{aligned}
$$

In (5.2) the max is taken for all numbers represented by Greek letters ranging between -1 and 1 . This example shows that $\lambda$ is not compatible with convolution in $l_{1} \otimes l_{1}$. We have not been able to determine whether in spite of this fact it is possible to turn $l_{1} \otimes_{\lambda} l_{1}$ into a Banach algebra under convolution and under a norm equivalent to $\lambda$.

6. Bases of tensor products of Banach spaces. We now examine the third question stated in the introduction. We consider two Banach spaces $E$ and $F$, which, for the present consideration, can be either real or complex. Suppose that $\Omega=\left\{x_{i}, x_{i}^{\prime}\right\}$, with $x_{i} \in E, x_{i}^{\prime} \in E^{\prime}$ is a biorthogonal system of $E$ defining a Schauder basis for $E$ and similarly for $\Lambda=\left\{y_{j}, y_{j}^{\prime}\right\}$ and $F$. In [2], we have shown that the biorthogonal set $\left\{x_{i} \otimes y_{j}, x_{i}^{\prime} \otimes y_{j}^{\prime}\right\}$ can be well ordered by means of a single index to form a biorthogonal system $\Omega \otimes \Lambda=\left\{u_{k} \otimes v_{k}, u_{k}^{\prime} \otimes v_{k}^{\prime}\right\}, u_{k} \in E$, $v_{k} \in F, u_{k}^{\prime} \in E^{\prime}, v_{k}^{\prime} \in F^{\prime}$, which defines a Schauder basis for both $E \otimes_{\lambda} F$ and $E \otimes_{\gamma} F$. These two tensor products had to be treated separately by methods based on their intrinsic properties. Now these two results can be obtained as consequences of the following general theorem because both $\lambda$ and $\gamma$ are uniform cross norms.

Theorem 6.1. Let $\Omega$ and $\Lambda$ define Schauder bases for $E$ and $F$, respectively, and suppose that $\alpha$ is a uniform cross norm of $E \otimes F$. Then $\Omega \otimes \Lambda$ defines a Schauder basis for $E \otimes_{\alpha} F$.

It should be remarked that the order given $\Omega \otimes \Lambda$ (the reader should consult [2] for details) is not completely arbitrary, as was amply demonstrated in [2]. There it was shown that even when $\Omega$ and $\Lambda$ define unconditional bases, the basis resulting from $\Omega \otimes \Lambda$ may not be unconditional.

\section{BIBLIOGRAPHY}

1. B. R. Gelbaum, Tensor products and related questions, Trans. Amer. Math. Soc. 103 (1962), 525-548.

2. B. R. Gelbaum and J. Gil de Lamadrid, Bases of tensor products of Banach spaces, Pacific J. Math. 11 (1961), 1281-1286. 
3. B. R. Gelbaum, Tensor products of Banach algebras, Canad. J. Math. 11 (1959), 297-310.

4. J. Tomiyama, Tensor products of commutative Banach algebras, Tôhoku Math. J. 12 (1960), 147-154.

5. R. Schatten, $A$ theory of cross spaces, Annals of Mathematics Studies No. 26, Princeton Univ. Press, Princeton, N. J., 1950.

6. A. Grothendieck, Produits tensoriels topologiques et espaces nucléaires, Mem. Amer. Math. Soc. No. 16, 1955.

7. J. Gil de Lamadrid, Measures and tensors, (to appear).

8. L. Loomis, Introduction to abstract harmonic analysis, Van Nostrand, New York, 1953.

UNIVERSITY OF MiNNESOTA

\section{AN INTEGRATION-BY-PARTS FORMULA ${ }^{1}$}

BY J. S. MAC NERNEY

Communicated by Walter Rudin, August 5, 1963

In 1914, W. H. Young [4] introduced a modification of the Riemann-Stieltjes integral which, for functions $F$ and $G$ defined on the real line with $G$ of bounded variation on each interval and $F$ suitably restricted, yields an additive interval function:

$$
\text { (Y) } \int_{a}^{b} F \cdot d G+(Y) \int_{b}^{c} F \cdot d G=(Y) \int_{a}^{c} F \cdot d G .
$$

In 1959, T. H. Hildebrandt [1] published a study of a certain linear initial-value problem involving these Young integrals, which extended some of the earlier results of $\mathrm{H}$. S. Wall and of the present author (see [2] for discussion and references). In 1962, there was discovered a connection between the Young integral and the interior integral as introduced by S. Pollard in 1920 [3], viz., the systems

$$
U(x)=U(c)+(Y) \int_{0}^{x} U \cdot d H \text { and } V(x)=V(c)+(I) \int_{x}^{c} d H \cdot V,
$$

with $H$ a function from the real line to a complete normed ring, are naturally adjoint to one another [2, p. 326]. Both integrals are to be interpreted as limits in the sense of successive refinements of subdivisions of the interval of integration.

Suppose each of $F$ and $G$ is a function from the real line to the complete normed ring $N$. If each of $F$ and $G$ is of bounded variation

\footnotetext{
1 Presented to the Society, July 18, 1963.
} 